\title{
Design and implementation of a control system for a deuterium pellet injector*
}

\author{
R. D. Burris, L. R. Baylor, D. E. Greenwood, and K. A. Stewart \\ Oak Ridge National Laboratory, P.O. Box Y, Oak Ridge, Ten- \\ nessee 37831 \\ (Received \\ CONF $-860324--15$ \\ DE $86 \quad 008503$
}

\begin{abstract}
The Oak Ridge National Laboratory is currently developing a Deuterium Pellet Injector for installation on the Tokamak Fusion Test Reactor (TFTR). This paper describes the design and development of a stand-alone data acquisition and control system for that device. Major elements of the hardware are an Allen-Bradley PLC 2/30 programmable logic controller, a MicroVAX-II computer using the VMS operating system, CAMAC data acquisition and communication equipment, and special-purpose controllers for temperature and for the sequencing of pellet firing valves. The PLC performs all actual control actions and acquires data pertinent to those actions. The MicroVAX receives the data acquired by the PLC, displays it for the operator, prompts for and processes requests for action from the operator, and informs the PLC of those requests. The primary purpose of this paper is to describe the software operating in the MicroVAX, including the system architecture, major tasks, and ancilliary and background tasks.
\end{abstract}

PACS numbers: 06

\footnotetext{
* Research sponsored by the Oflice of Fusion Energy, under Contract No. DE-AC05-840121400 with Martin Marietta Energy Systems, Inc.
} 


\section{INTRODUCTION}

The development of pneumatic pellet injectors for fueling plasma fusion devices has been under way at ORNL since $1976 .{ }^{1}$ Until recently, pellet injection experiments had been carried out on tokamaks at ORNL and other laboratories with minimal controls on the injection system. However, with the current generation of plasma devices, it is necessary both to have remote control of all aspects of the pellet injector's operation and to record important pellet parameters such as speed and size. Independent pellet injector controls are also desirable so that the injector system can be fully tested before installation on a particular plasma device.

A control and data acquisition system has been designed at ORNL which can be used with ORNL pellet injectors on any tokamak device without major modification. The system was first implemented for the Repeating Pneumatic Injector (RPI) ${ }^{2}$ that was installed on the Tokamak Fusion Test Reactor (TFTF) at the Princeton Plasma Physics Laboratory (PPPL). A monitoring-and-control system for the ORNL 
Radio Frequency Test Facility (RFTF) was subsequently implemented based upon the RPI design. ${ }^{3}$ The system described in this paper has incorporated the good elements of each system and elaborated upon them in several ways.

\section{CONFIGURATION}

A block diagram of the control and data acquisition system as it will be installed on TFTR is shown in Fig. 1. A MicroVAX II minicomputer is the interface between the user/operator and the system. The computer utilizes CAMAC equipment to perform data acquisition and to communicate with the cryogenic temperature controllers (Lakeshore model DRC-82Cs), the pellet-firing sequencer (designed and built by PPPL), and an Allen-Bradley PLC 2/30 programmable controller.

The PLC performs all actual interlock checking and control except functions associated with the pellet firing sequencer. Operator requests for control actions are made via software on the MicroVAX, but those requests are simply passed on to the PLC for action. 


\section{LOCAL CONTROLS}

Local operation of the injection system is possible without interfacing with the MicroVAX. The PLC has an intelligent panel system that has a keypad and lighted pushbutton modules programmed from the PLC that can be used to enter setpoints and control output devices. The temperature controllers each permit entry of setpoints on their front panels. The pellet firing sequencer has thumbwheel switches for loca] entry of each of its setpoints.

\section{NORMAL CONTROL SYSTEM}

The Deuterium Pellet Injector (DPI) is normally operated remotely - that is, the operator views screens and makes requests at a control station physically distant from the injector. There are two major categories of tasks which make this operation possible - one that consists of three communications tasks and another consisting of the operator interface tasks.

Communication between the different tasks occurs in several ways, but the primary mechanism is the use of shared global common memory. There are two such shared commons - 
DPISTATUS and DPICONTROL. The former

is used to contain current status information about all elements of the system, while the latter contains all current setpoint values and other control-related entities.

\section{A. Communications Tasks}

There are three major control devices in the system, and there is a corresponding VAX communication task for each. The tasks are named TEMPS, PLC, and SEQUENCER.

The temperature controllers are GPIB (IEEE-488 Bus) devices which communicate with the VAX TEMPS task via a GPIB-ASCII converter and a CAMAC RS232 module. The TEMPS task sends setpoints to each controller, queries each about its current setpoints, and obtains the current temperature sample from each one every second.

The PLC task also uses a CAMAC RS232 module to communicate with a corresponding PLC ASCII module. The PLC sends three strings of status information to the VAX each second. One string contains the status of approximately 200 Boolean entities, and 
the other two include the binary-coded-decimal (BCD) values of approximately 50 entitics.

When the operator makes a command request, the VAX PLC task sends one of two strings to the PLC. If the operator requests a change in the state of a Boolean entity, a string of all such Boolean request bits, with the appropriate one set true, is sent to the PLC. If the request is to change a setpoint, a set of Booleans to identify the proper setpoint variable, with the appropriate one set true, is sent to the PLC and followed, in the same string, with a single BCD setpoint value. Each of the strings actually contains two copies of everything so that the PLC ? can, through an exclusive-or operation, verify that no errors have occurred in transmission.

The SEQUENCER task communicates via three CAMAC modules to a specially-designed firing sequencer built by PPPL. The VAX task sends blocks of setpoints (BCD) to the sequencer and reads the contents of its registers for verification. When triggered by the TFTR timing system, the firing sequencer triggers the solenoid valve power supplies that fire the appropriate pellets. The outputs from the pellet-firing 
sequencer also trigger the transient recorders that are used to collect the pellet diagnostic data.

\section{B. Operator Interface}

The operator interface consists of three display devices and their software: a color mimicpanel display (with an associated trackball), a menu-display terminal, and a graphics terminal for analysis. System status is displayed primarily on the color mimic-panel display, but a few selected points are also shown on the menu terminal. When the state of some device is to be changed, the operator uses the trackball to move the mimic-panel-display cursor to the appropriate entity and then pushes a button to notify the VAX that an action is to be taken. When typed information is to be supplied, it is entered into a field of a form displayed on the menu terminal using the keyboard of that terminal.

\section{Mimic-Panel System}

The mimic-panel system displays one of eight mimic panels representing different subsystems of the pellet injector. The fueling gas and propellant gas systems are shown on one panel each, while the vacuum and cryogenic systems each 
require two pages to represent them. There arc also two pages for pellet handling (the extruder and cold-wheel systems), one for ORNL testing and one for system operation. Each panel contains a five-line common header section containing mimic-panel and menu selections, operational modes, and system status.

The MIMIC task controls and updates these displays by reading information from the DPISTATUS shared global common, converting this information to a display format, and sending the results to the display driver device (Kinetic Systems 3242) which updates the display.

\section{Menu System}

It is through the menu system that information is entered by the operator. This information, such as setpoints or gas species, is typed on the keyboard of the menu display terminal after the operator has chosen an activity from the form currently displayed. There are nine forms in the system: one each for temperature, pressure, and extruder setpoints; one for firing sequencer parameters; and forms for display of error counters, for control of automatic processing, for digi- 
tizer parameter settings, for clock parameter settings, and for the choosing of forms.

The menu system is also used for the display of status data of global interest such as critical temperatures and pressures. Those data appear on each form and are updated each second.

The menu task has three unique features it has a keyboard input device, it has a display screen, and it is active each second. This combination makes the menu task the only candidate for the implementation of control-related activities, such as asking the operator to verify that the last request was accurate or identifying and informing the operator of error conditions. These functions are included in the ONCE-PERSECOND subroutine of the menu task.

\section{DATA ACQUISITION}

CAMAC digitizers are used to sample pressure transients, speed diode signals, and $\mathrm{H}$-alpha light emissions from the pellet/plasma interaction region. These digitized signals are stored in digitizer memory until after the tokamak shot, then dumped through the VAX to disk data sets for later analysis. In addition, data that were 
received from the PLC and TEMP tasks are kept (vin a TREND task) in a trend data set for later analysis.

\section{DATABASE}

One of the new features in the DPI software is the use of the System 1032 data base management product. There are datasets pertaining to the PLC, to the DPISTATUS shared global common, to the DPICONTROL common, and to the mimic-panel system.

In order to use a shared global common, each FORTRAN routine which references the common must describe it. $\mathbf{S 1 0 3 2}$ report-writing command files are used to generate the files defining the DPISTATUS and DPICONTROL commons (including the COMMON statement and the type statements, such as INTEGER* 4 and $\mathrm{REAL}^{*}$ ) from the datasets of the same name. The generated files are INCLUDED in FORTRAN programs to define the relevant variables.

When using shared global commons, it eases debugging and testing to have tasks that will initialize, dump, and manipulate those commons. Both datasets are used, via additional S1032 
report-writing command files, to generate the FORTRAN programs for initializing and dumping the commons and to generate additional INCLUDE files for the testing programs. In similar manner, the PLCVAX dataset is used to generate the subroutines by which the VAX decodes strings received from the PLC (placing the data in the proper variable of DPISTATUS common) and the subroutines that ase data in DPICONTROL common to construct strings that are sent to the PLC.

System changes are handled in the following manner. When a new entity is added to the system, an item is added to the PLCVAX dataset. The command files that generate reports on that dataset and that generate string construction and decoding routines are invoked and the resulting files sent to the MicroVAX. Such changes also often involve either the DPISTATUS or DPICONTROL datasets, so corresponding changes are made to them (verifying the changes against those in the PLCVAX dataset via the MAP cormmand). Then new INCLUDE files are generated and sent to the MicroVAX along with new DUMP and CLEAR utilities. The new 
INCLUDEs are then used in recompilation of all relevant routines. There are command files associated with each dataset that invoke all required tasks associated with a change in the contents of the dataset. Similarly, there are command files on the MicroVAX to incorporate new INCLUDE files in each affected routine.

\section{ACKNOWLEDGMENTS}

The authors wish to acknowledge the efforts of S. L. Milora, S. K. Combs (both of ORNL), and G. L. Schmidt (PPPL) for the specification of the system requirements. D. R. Overbey put the VAX hardware and software together, and E. T. Blair developed and debugged the CAMAC software driver. Jim Snyder (PPPL) wrote the analysis tasks that massage the data acquired by this system and was responsible for the RPI software during its year of operation on TFTR. C. R. Foust, R. C. Shanlever, D. O. Sparks and A. Waycaster constructed and helped check out the injector at ORNL.

Research was sponsored by the Office of Fusion Energy, under Contract No. DE-AC05- 
84OR21400 with Martin Marietta Energy Sys-

t.cuns, Inc. 


\section{REFE ? ENCES}

'S. L. Milora, J. Fusion Energy, 1, 15 (1981).

${ }^{2}$ L. R. Baylor, R. D. Burris, D. E. Greenwood, and K. A. Stewart, "Design and Implementation of a Control and Data Acquisition System for Pellet Injectors," in Proceedings of the 11th Symposium on Fusion Engineering, November 18, 1985, Austin, Texas.

${ }^{3}$ K. A. Stewart, R. D. Burris, J. B. Mankin, and D. H. Thompson, "The Computer Control and Data Acquisition System for the R.F. Test Facility," in Proceedings of the Sixth Topical Conference on High Temperature Plasma Diagnostics, March 9-13, 1986, Hilton Head, South Carolina. 


\section{FIGURE CAPTIONS}

FIG. 1. DPI stand-alone controls hardware arrangement. 
DPI STAND-ALONE CONTROLS HARDWARE ARRANGEMENT

ORNL-DWG 86-2024 FED

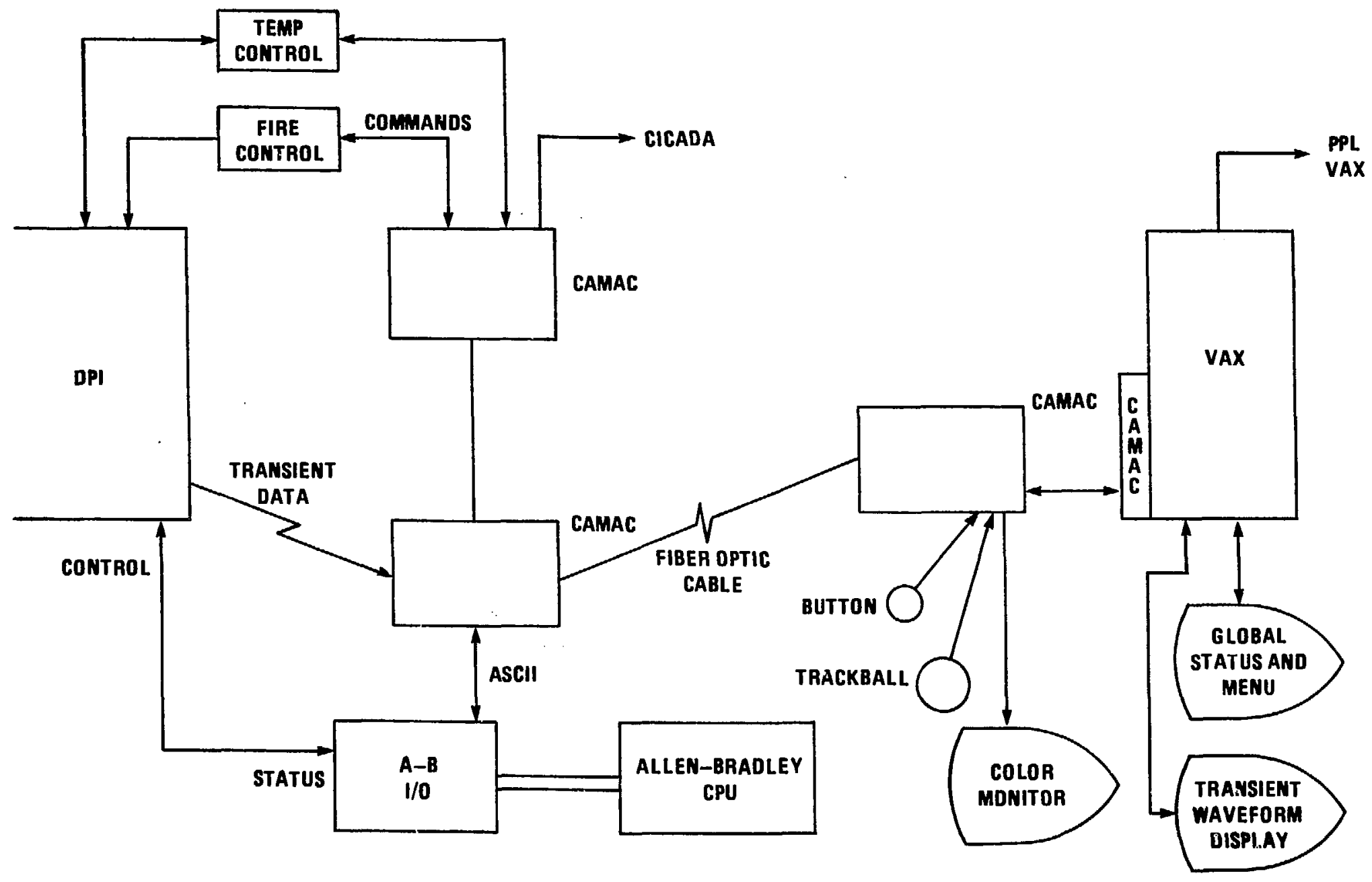

